\title{
PROFILES OF OUTPATIENT SPEECH-LANGUAGE THERAPY AND AUDIOLOGY CLIENTS AT A TERTIARY HOSPITAL IN THE WESTERN CAPE
}

\author{
Wendy Overett and Harsha Kathard
}

Division of Communication Sciences and Disorders, University of Cape Town

\begin{abstract}
This study described trends in the demographic, diagnostic, and attendance variables of first-time clients who attended the SpeechLanguage Therapy and Audiology outpatient department (OPD) at a tertiary hospital in the Western Cape over a five-year period (19992003). A retrospective, descriptive survey was conducted and data were collected from hospital records. The data were coded, analysed descriptively and represented in tables, figures and graphs. The diagnostic categories of hearing loss and voice disorder made up the highest percentage of referrals. The largest percentages of clients were referred from the Ear, Nose and Throat (ENT) Department at the study hospital, followed by the education sector. Demographic trends indicated that the highest percentage of clients attending the OPD was in the age category of 7-12 years, that similar percentages of male and female clients attended the OPD, and that English was the language that a client was most likely to speak. Geographically, the highest percentage of clients attending the OPD was from the Klipfontein region. Attendance data revealed that approximately $70 \%$ of clients attended OPD appointments within a three-month time period and attended no more than two appointments. Percentage of appointments attended decreased with increasing numbers of scheduled appointments. Implications for research and service delivery are discussed.
\end{abstract}

Key words: Speech-Language Therapy and Audiology services, healthcare, service delivery, client profiles, attendance

\section{INTRODUCTION}

This study aimed to describe the client population profiles of those attending the speech-language therapy and audiology outpatient department (OPD) at a tertiary hospital in the Western Cape in South Africa over a five year period (1999-2003). To achieve this aim, the demographic, diagnostic and attendance data of a study sample were determined. The impetus for this study arose from difficulties the researcher experienced while attempting to engage with service planning for Speech-Language Therapy services in a post-apartheid era at a tertiary hospital in the absence of knowledge of descriptive client data.

In the South African public sector, SLT \& Audiology services háve traditionally been lociated in both the health and education sectors. In the health sector these services have been considered as part of allied health service delivery with emphasis on rehabilitation (Allied Health Professionals Technical Committee, 2004). Although health services are essential, they fail to reach many people in South Africa due to a lack of resources. Historically, health service delivery in South Africa has been shaped by the medical model and complied with apartheid ideology (Bhagwanjee \& Stewart, 1999; Hall, Haynes \& McCoy, 2002). Public health services were characterised by racial and geographical disparities, fragmentation and duplication of services and were hospital-centred with minimal emphasis on Primary Health Care (PHC). The combined influences of the medical model and apartheid ideology resulted in an inequitable provision of services in terms of accessibility, appropriateness, funding and co-ordination across the variables of race, class, gender and level of urbanisation (Bhagwanjee \& Stewart, 1999). The healthcare system was divided, inefficient and grossly inequitable (Hall et al., 2002).

Speech-Language Therapy (SLT) and Audiology Services in South Africa have been shaped by such socio-political contextual realities and are also grossly inequitable (Pillay, Kathard \& Samuel, 1997). Services in South Africa have been biased toward providing a better quality service to a White, middle class, English and Afrikaans first language speaking population, whilst pro- viding a poorer service to a Black African first language speaking clientele (Pillay, 1996 cited in Pillay et al., 1997). Public sector SLT \& Audiology services have historically been based in hospitals and special schools, with minimal service delivery at community level. Similar to the national profile, services in the Western Cape have been urban-based with few Speech-Language Therapists and Audiologists working in rural areas beyond the Cape Metropole. Health sector provision of SLT \& Audiology services have been limited to services at tertiary hospitals, with little available at primary or secondary levels in the Western Cape (Allied Health Professionals Technical Committee, 2004), thus limiting access and availability of services to the majority of the population.

The current service delivery situation is untenable given the prevalence of disability in the Western Cape. The data from the 2001 Census (Statistics South Africa, 2004) regarding the numbers of people with communication, hearing or multiple disabilities point to the need for SLT \& Audiology services in the City of Cape Town and in the Western Cape. The need for accessible services, coupled with appropriate planning, and importantly the knowledge to assist such planning, is essential to meet the service delivery needs of this population.

Over the last decade, systemic changes have taken place within the health system which have had a bearing on SLT \& Audiology services. Subsequent to South Africa's first democratic elections in 1994, the National Department of Health set about transforming and restructuring the healthcare system (Hall et al., 2002; Forman, Pillay \& Sait, 2004). These changes included a policy shift to a primary health care (PHC) approach within a district health system (DHS), with intent to realise a social model of health. The aim of such transformation has been to improve the quality of care provided and create a more equitable service. There is a critical need for relevant knowledge to inform such planning processes. In order to ensure cost-effective improvement and development of existing health services, the efficacy of health programmes needs to be validated through research showing that such services are necessary, appropriate and accessible, within 
specified contexts (Bhagwanjee \& Stewart, 1999)

Several studies within the SLT \& Audiology profession, have examined client profiles to facilitate service planning. Internationally studies by Broomfield and Dodd (2004); Edwards, Cape and Brown (1989); Enderby and Davies (1989); Enderby and Petheram (2000); Heron (2001); and Petheram and Enderby (2001) have provided critical data to influence service planning. In South Africa a limited number of studies have examined client data in different contexts (Klop, 1998; Schneider, 1992 and Swanepoel, 2005).

Klop (1998) conducted a study in the area of quality management in a private healthcare practice in Cape Town. The outcomes of the study were intended to help implement quality management programmes. Client profiles in terms of disorders, age, geographical location, gender, home language and referral agents were compiled from 197 clients seen by the researcher in her practice from 1994 to 1996 . She found that the majority of clients attended for language disorders, followed by stuttering, almost half were between three and six years of age, $63 \%$ of the clients were nale, almost three quarters of her clients were Afrikaans-speaking and most of the clients were drawn from an area within a $10 \mathrm{~km}$ radius of the practice. Her clients were referred from other healthcare professionals, educators and former clients. From a smaller group of clients she found that only two out of 64 clients withdrew from therapy.

Schneider (1992) collected data from records, reports and case files to determine the nature and prevalence of communication disorders seen in six hospitals in Gazankulu. Communitybased speech and hearing workers were then interviewed about their work situation, organisation of their time and intervention strategies used with communicatively disordered people in order to evaluate the efficacy of their work. The most common disorders seen at the hospitals in Gazankulu by these speech and hearing workers were hearing disorders.

Swanepoel (2005) conducted an exploratory descriptive study which critically described an infant hearing screening programme conducted at two maternal and child health clinics in Hammanskraal. Quantitative and qualitative methods for data collection were used His study showed poor follow-up return rates to the clinic.

In South Africa, apart from these studies by Klop (1998), Schneider (1992) and Swanepoel (2005) there is limited data to contribute to service planning. The unique characteristics and challenges of developing countries demand that contextual and tocal research be done in conjunction with international studies (Swanepoel, 2005).

Speech-Language Therapy research in the United Kingdom has emphasised the importance of ongoing gathering information on services and client populations for Speech-Language Therapy services. As with South Africa, inequalities in services for the speech and language impaired in the United Kingdom's national health service have been documented (Enderby \& Davies, 1989). If there is to be efficient planning in healthcare it is important that this process be informed with regard to the size and needs of the population to be served (Enderby \& Davies, 1989). The collation of client data profiles may serve in questioning some of the basic premises regarding provision of services and should lead to consideration of alternative forms of provision which meet the needs of the speech and/or language disabled and hearing impaired popuTation (Enderby, 1989).

To monitor equity concerns in South Africa, comparative data are necessary to understand the multifaceted nature of client profiles which include race, age, gender, urban/rural location and socio-economic status (Ntuli \& Day, 2004). The first steps however, are to collect data from current service sites, as was the intention of the present study. The demographic, diagnostic and attendance data of clients receiving services at a tertiary level of healthcare could be valuable in planning equitable and appropriate services.

Reliable data concerning the types of speech, language and hearing disorders referred, age and sources of referral, and the effect of cultural and socio-economic profiles of the population on referral patterns are vital for planning services (Broomfield \& Dodd, 2004). Comparison of records may show service trends, admission patterns and familial trends (Lubker \& Tomblin, 1998) over time. It was important to collect data gathered over time so that changes in service provision could be examined with the purpose of informing debate and planning (Petheram \& Enderby, 2001). The compilation and analysis of data, timeous reporting and use of consistent up-to-date health information, are all key aspects of healthcare planning and management. The lack of information regarding the health sector has contributed significantly to the slow process of transforming the health system (Ijumba \& Day, 2004). Thus the results of this study, with its focus on demographic, diagnostic and attendance data, has potential to influence service delivery changes with regard to SLT \& Audiology services.

\section{METHODOLOGY}

\section{Aim}

This study aimed to describe trends in the demographic, diagnostic, and attendance variables of first-time clients who attended the SLT \& Audiology outpatient department (OPD) at the tertiary hospital being studied over a five year time period (19992003).

\section{Study design}

A retrospective descriptive survey was conducted, to collect demographic, diagnostic and attendance data of a sample of clients.

\section{Sample}

The study population included all the hospital records of new clients who had attended the speech-language therapy and audiology OPD of the tertiary hospital under study during a 5 year period from January 1, 1999 to December 31, 2003. This period of time was selected as data were readily available and would give a sufficiently large number of records for trends over the years to be examined. Due to the large number of clients in the population $(2,819)$, a sample of one-third of the population data were drawn, using a stratified random sampling procedure (Katzenellenbogen, Joubert \& Abdool Karim, 1997), stratified according to the year in which the client was first seen. The sample consisted of 929 clients, on which the analysis was conducted.

\section{Data collection and analysis}

The clients' names were retrieved from the client registers and record cards. The data collection form, devised by the researcher, was then utilized to capture the data from the hospital records (Appendix A). Data were electronically captured onto a spreadsheet as raw data and then entries were coded by the researcher. In instances where data were missing on the form (where it was not indicated as missing in the records), the records were 
reviewed again by the researcher to retrieve this information. After the data had been entered into the computer and checked, client names and folder numbers were removed to ensure anonymity of the clients. Attendance data were then converted to number of appointments attended, time frame over which these appointments were attended and the percentage of appointments attended. The coded data were transferred from the spreadsheet into a computerdriven statistical package (Statistical Package for the Social Sciences) (SPSS Inc, 1995) and frequency and percentage distributions as well as contingency tables were generated.

In instances where a data collection form had missing data, after being re-checked by the researcher, information which was unavailable, was categorised as such. If a record for a client had missing data for two or three of the three diagnostic variables, the record was excluded from analysis of diagnostic variables and attendance variables, but was still included in the analysis of demographic variables. In instances where there was no clear attendance data for two or more attendance variables, that client was excluded from the analysis of attendance information. However, if only one of these variables was missing, the data were still included in the overall attendance analysis.

Due to the descriptive nature of this study, analysis of variables was conducted via descriptive statistics (Hite, 2001), as used in other similar studies (Enderby \& Petheram, 2000; Petheram \& Enderby, 2001). Frequency and percentage distributions were used to describe the demographic, diagnostic and attendance data. Frequency data were converted into percentage data for each year and for the total sample. Contingency tables (cross tabulation) were used to show relationships between variables. Inferential statistics were not used as this form of data analysis is not appropriate for a descriptive study (Hite, 2001).

\section{Reliability and validity}

Reliability of the data collection procedure was ensured by defining the exact methods of measuring and checking of work (Katzenellenbogen, et al., 1997). In this study a standard, reliable data collection form (Appendix A) was used to collect data. Crosschecks of information from the hospital record sheet to the SLT \& Audiology folder also enhanced the reliability of the data collection process.

Areas of concern for validity included the reality that hospital records are generally produced for clinical, administrative or monetary ends rather than research purposes (Abramson, 1990) and that different clinicians may have used different terminology when describing diagnoses. Terminologies were therefore examined and then grouped in broad categories. Data collection was verified by the researcher as it was entered into the computer.

Instrument, observer and client variations can be evaluated by repeating measures of a sub-sample of the study sample (Katzenellenbogen, et al., 1997). In this study a random sample of ten percent of the clients from each year was selected for recollection of data $(n=93)$ by an independent researcher. The data were then coded and the codes were compared to those found for the same clients by the researcher. There was $95 \%$ agreement across all the variables, indicating good inter-observer reliability.

A large sample was chosen to enhance the validity of the study as confidence intervals become narrower as sample size increases (Katzenellenbogen, et al., 1997). The data were analysed quantitatively using SPSS to ensure the data were analysed in a consistent manner thereby ensuring valid and reliable results. The results were interpreted by the researcher who also engaged with a process of peer review to enhance the quality of the interpretation. Ethical considerations

The head of the speech-language therapy and audiology department, the medical superintendent of the tertiary hospital being studied, and the ethics committee of the University of Cape Town were consulted with regard to gaining access to the hospital files. It was not, however, possible to gain consent from the clients, due to potential difficulties locating clients and the number of records being reviewed. However, confidentiality was strictly maintained by removing any identifying information after the data had been checked and coded. Access to the files may be considered ethical on the grounds that there was no risk of harm to individuals, there was potential public benefit, and investigators' protected the confidentiality of the individuals whose data they studied (Council for International Organisations of Medical Sciences, 1991). The community will stand to benefit from this research if changes, taking into account the findings of this study, are made within the healthcare setting with regard to equity in service provision and aligning the services to the needs of the population.

\section{RESULTS AND DISCUSSION}

The number of clients varied each year ranging from 178 to 197 clients, with a total sample size of 929 clients on which these results are based. The results and discussion are presented in relation to the aims of the study. The total number of clients varied across the aims because of missing information as explained in the methodology. The quantitative results are represented in tables and graphs and the main trends are described and then discussed.

\section{Diagnostic data}

\section{Speech-Language Therapy and Audiology Diagnosis}

In this study recorded reported medical diagnoses have been managed as inter-related diagnostic categories with SLT \& Audiology diagnoses. The percentage of clients within each SLT \& Audiology diagnostic category is presented in Figure 1. Speech-Language Therapy and Audiology diagnostic categories of those attending the OPD appeared to remain fairly consistent over the five-year period, with minor variations across the years.

The common diagnoses of those attending the SLT \& Audiology OPD included hearing loss $(\mathrm{n}=255 ; 30 \%)$, voice disorders $(\mathrm{n}=177 ; 21 \%)$ and neurogenic communication disorders. The types and percentages of diagnoses remained relatively similar across the five years.

There are several significant issues surrounding the types of disorders seen at the SLT \& Audiology OPD and the percentage of clients attending the OPD for the management of each disorder (Figure 1). The reason for the high percentages of clients attending the OPD as a result of a hearing loss or voice disorders, may be due to the fact that this SLT \& Audiology OPD is situated in a tertiary hospital, with specialized equipment and facilities suitable for assessing and treating these disorders.

Due to the fact that hearing loss and voice disorders, as well as neurogenic communication disorders (for which the third highest percentage of clients attended the OPD) are medically based and are treated by the medical profession in conjunction with Speech-Language Therapists and Audiologists, clients may be likely to attend this hospital which offers both services. Hearing loss, voice and neurogenic communication disorders are often acquired and therefore there is a 'loss' of function, making the disorder noticeable and igniting a desire or need to restore lost function. 


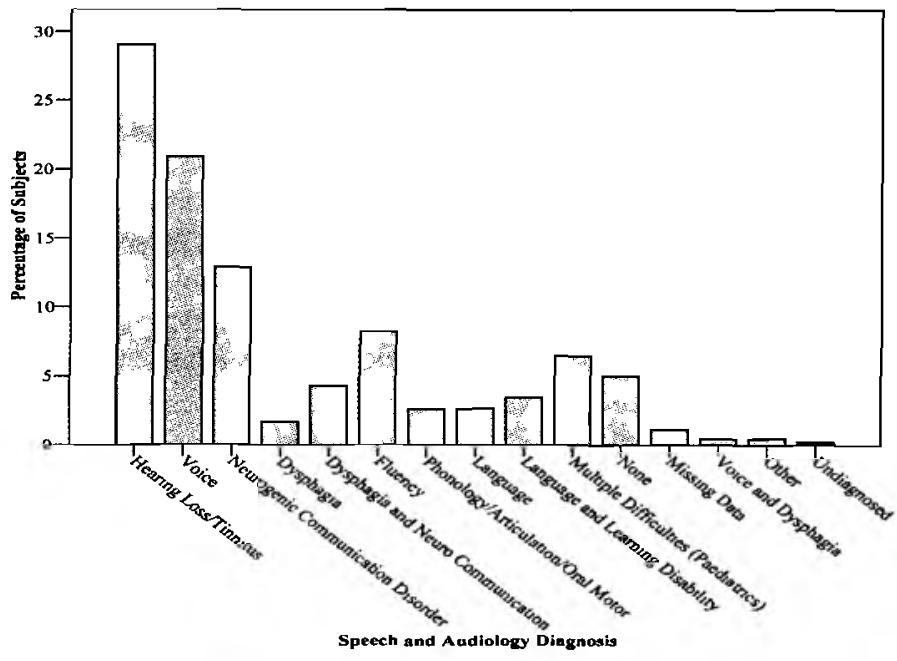

Figure 1: Percentage of clients per Speech-Language Therapy and Audiology diagnostic category who attended the Speech-Language Therapy and Audiology OPD at a tertiary hospital in the Western Cape from 1999-2003 $(n=859)$.

The proximity and connections to the ENT department, who made the largest percentage of referrals to the SLT \& Audiology OPD, may also account for the high percentages of clients who attended the OPD as a result of a hearing loss or voice disorder.

The highest percentages of clients were diagnosed with hearing losses, voice disorders, neurogenic communication disorders or fluency disorders, which are 'noticeable' disorders and thus easier to detect than less 'visible' disorders (McLaren, Solarsh \& Saloojee, 2004) and are likely to impact on everyday life. Less 'visible' disorders, such as a language impairment, may not be as easily noticeable in everyday situations and thus not as easily detected. These less 'visible' disorders are then not as likely to be referred to SLT \& Audiology services. It is also possible that because the Department is actually referred to as a "speech therapy" rather than "speech-language therapy", it may not be obviously associated with managing language disorders, a reality that the profession has experienced internationally.

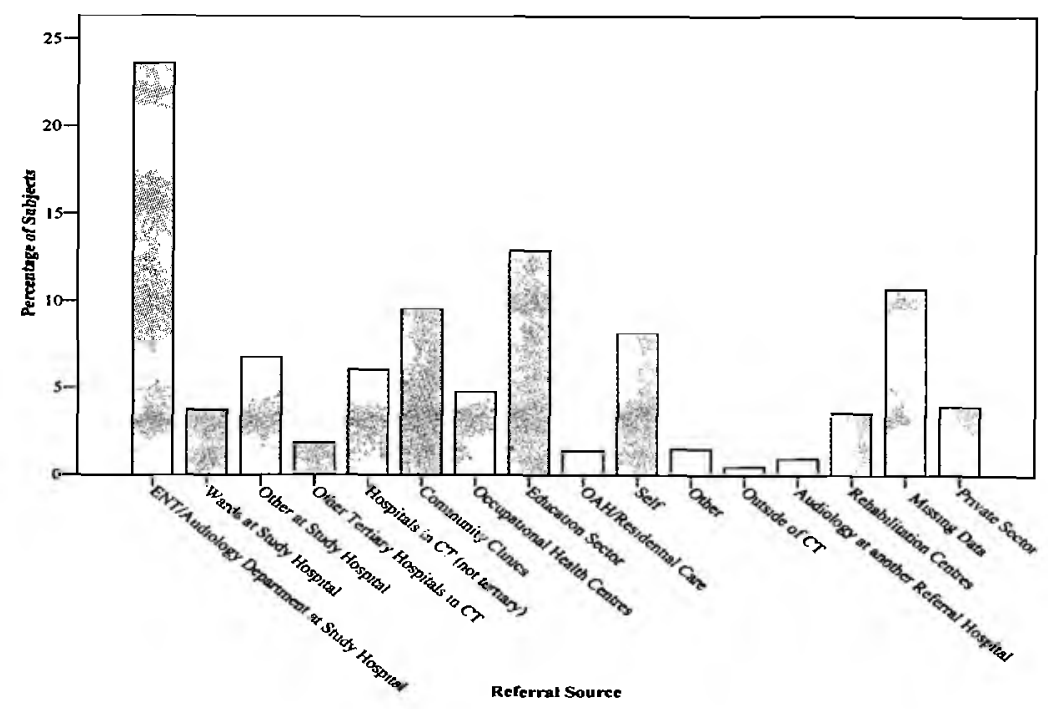

Figure 2: Percentage of clients per referral source who attended the Speech-Language Therapy and Audiology OPD at a tertiary hospital in the Western Cape from 1999$2003(n=859)$.

\section{Referral Sources}

The results for referral sources appear in Figure 2. From the year 2000 onwards there was an increase in referrals from community clinics and from wards at the study hospital.

When examining individual categories of clients with a specific disorder or from a specific referral source, particular trends were found. These results are based on the clients within a specific category only and thus the percentages and numbers are based on these individual categories and not on the full sample. The majority of clients who attended the SLT \& Audiology OPD as a result of a voice disorders $(n=137 ; 76 \%)$ were referred from the ENT department. Fifty percent $(n=41)$ of referrals from community clinics, $80.5 \% \quad(n=33)$ of referrals from occupational health centres and $83 \%$ $(n=10)$ of referrals from old age homes or residential care were referred to the SLT \& Audiology OPD as a result of a hearing loss. The majority of the referrals to the OPD from wards were for dysphagia, neurogenic communication disorders or a combination of these $(n=31 ; 97 \%)$. The education sector referred $50 \%(n=28)$ of paediatric clients with multiple difficulties to the OPD.

Referral sources (Figure 2) would also have shaped the client diagnostic profiles discussed above. As mentioned, the ENT department made a high percentage of referrals to the SLT \& Audiology OPD. This trend is understandable in light of the discussion around the types of disorders seen most frequently at the OPD. The second major referral agent to the OPD was the Education Sector. This situation may be attributed to the limited SLT \& Audiology services within mainstream schools (F. Lewis, personal communication, February 18, 2005), resulting in referrals to the Health sector.

\section{Demographic data}

\section{Age Groups}

Age group results, presented in Table 1, illustrate that the largest percentage $(n=165 ; 18 \%)$ of clients who attended the OPD were within the age group of 7 12 years.

The following results are based on sub-categories of clients. Clients attending the OPD with a hearing loss or tinnitus covered the full range of ages, although the majority of these clients were between 30 and 79 years of age $(n=181$; $72 \%$ ). The majority of clients who attended the SLT \& Audiology OPD as a result of a voice disorder were between 30 
and 49 years of age $(n=98 ; 54 \%)$. Clients with a neurogenic communication disorder spanned a wide range of ages, although the highest percentage fell within the age group of $50-59$ years $(n=27$; $24 \%$ ). The majority of clients who attended the OPD as a result of dysphagia were between 50 and 69 years of age $(n=10 ; 67 \%)$, while clients with both dysphagia and a neurogenic communication disorder were slightly older, between 60 and 79 years $(n=23$; $62 \%$ ). The majority of clients presenting with fluency disorders, however, were under 18 years of age $(n=64 ; 90 \%)$. A similar trend occurred for clients attending as a result of a phonology/ articulation/oral motor disorder, language disorder, language and learning disorder or multiple difficulties although the majority were under the age of $12(n=20 ; 87 \%)$. While the highest percentage of clients attending as a result of language disorders was in the under six year age group $(n=15 ; 65 \%)$, children attending the SLT \& Audiology OPD with language and learning disorders or multiple difficulties had a greater percentage in the 7-12 year old category $(n=25 ; 83 \%$ and $n=35 ; 62.5 \%$ respectively).

Despite the high number of clients with a hearing loss or voice disorder attending the OPD (which in this study were found predominantly in the adult population), the majority of the caseload attending the SLT \& Audiology OPD was found to be between 7 and 12 years of age (Table 1). Of this age group only a minority attended the OPD for a hearing loss or voice disorder. However, this age group had the majority of the clients with fluency disorders, phonology/articulation/oral motor disorders, language and learning disorders, as well as those with multiple difficulties. A reason for this high percentage of 7 to 12 year olds attending the OPD under study in relation to the under six population, may be due to the close proximity of another tertiary hospital, which specializes in paediatric intervention. The high percentage of 7 to 12 year olds attending the OPD (who are primary school age children) alludes to the lack of services within the education system, who were also the second highest referral agent. Despite the inclusive education policy intention regarding inclusive education (Department of Education, 2001), there seems to have been minimal support for learners with communication difficulties in mainstream schools in the Western Cape.

The researcher has also perceived a general lack of knowl-

Table 1: Number and percentage of clients per age group who attended the Speech-Language Therapy and Audiology OPD at a tertiary hospital in the Western Cape from 1999-2003 $(\mathbf{n}=910)$

\begin{tabular}{|l|l|l|}
\hline \multicolumn{1}{|c|}{ Age } & \multicolumn{1}{c|}{ Number } & \multicolumn{1}{c|}{ Percent } \\
\hline $0-6$ years & 69 & 7.58 \\
$7-12$ years & 165 & 18.13 \\
$13-18$ years & 39 & 4.29 \\
$19-29$ years & 79 & 8.68 \\
$30-39$ years & 118 & 12.97 \\
$40-49$ years & 115 & 12.64 \\
$50-59$ years & 107 & 11.76 \\
$60-69$ years & 101 & 11.10 \\
$70-79$ years & 87 & 9.56 \\
$80+$ years & 30 & 3.30 \\
Total & 910 & 100 \\
\hline
\end{tabular}

edge amongst educators, many health professionals and the public, of SLT \& Audiology services, resulting in 'late' referrals of individuals. Anecdotal parent reports have indicated that parents who had voiced their concerns regarding their child's delayed language development have been told by health professionals 'not to worry' or that their child will 'grow out of it'. Thus, instead of the child receiving early intervention, intervention was only available upon entering school where there are high demands on communication skills and the child's difficulties begin to impact on academic performance. This scenario again relates to the 'visibility' of certain disorders (McLaren et al., 2004), as these language and learning disorders are often only detected in an academic environment and are not immediately obvious in everyday situations (Das, 2001).

Examination of the results pertaining to the adult population attending the Speech-Language Therapy and Audiology OPD showed that there was a slight decrease in percentage of clients attending the OPD within each 10 year category from 30 to 69 years, with a slightly larger decrease in therapy attendance at 70 to 79 years and a large decrease in therapy attendance in the over 80 year old category. An explanation for the greatest number of adults attending the OPD being between 30 and 49 years of age may be due to the large number of clients attended the OPD as a result of a voice disorder and the fact that the majority of clients with voice disorders were in this age group.

This finding of a decrease in therapy attendance amongst clients with increasing age, however, seems to follow a general trend in rehabilitation, where services are less accessible and available to the older population. Although disability is more prevalent in the older population, it seems that older adults are unable to access hospital based outpatient services. Blake (1981) in the United States of America, claims that in terms of age group, participation in the rehabilitation service seems to be inversely related to the need for such service. Rehabilitation may be more of a priority for younger people, or services may be more accessible to them, than for the older population. Rehabilitation has previously been driven both philosophically and financially by the goal of restoring individuals to productive employment, resulting in better opportunities for younger clients, although this is now changing to include a focus on older clients (Raia, 1992). The motivation to attend rehabilitation may be greater for the younger adults due to their need to regain employment, as well as to pursue social goals. A study at Groote Schuur Hospital in the Western Cape, South Africa (Whitelaw, Meyer, Bawa \& Jennings, 1994) confirmed this trend and reported that greater numbers of stroke clients under the age of 65 years old, who had been inpatients, were referred (as outpatients) to Physiotherapy and Occupational Therapy services, while fewer clients above the age of 65 were referred. Furthermore, a greater number of the younger adults presented for and received therapy than older adults. Another study in the Western Cape, examining rehabilitation services at Bishop Lavis Rehabilitation Centre, documented that $50 \%$ of the population attending these services were 60 years old or younger (Rhoda, 2001).

In light of the above discussion regarding services for different age groups of clients, the current study revealed that a large percentage $(n=71 ; 64 \%)$ of clients with a neurogenic communication disorder (without dysphagia) were under the age of 60 years. However, $70 \%(n=26)$ of those with both dysphagia and a neurogenic communication disorder were over the age of 60 . This possibly indicates a greater degree of disability in the older age groups, where multiple disabilities are perhaps more likely. This trend of multiple disabilities in the older population may also explain the reduction in the number of these older clients attending outpatient rehabilitation. Elderly clients may not only be more 
affected by a stroke, but may also have more problems reaching therapists than younger clients (Whitelaw et al., 1994). There is also greater attrition of older clients than younger clients due to stroke (Whitelaw et al., 1994).

\section{Gender}

Slightly more than half of the sample who attended the OPD was male $(n=470 ; 52 \%)$. More females than males presented with voice disorders, language disorders or no specific Speech-Language \&/or Audiology diagnosis.

It was unexpected to find similar percentages of male and female clients attending the OPD, as the belief was that there would be many more males than females as found in other studies (for example Broomfield \& Dodd, 2004; Enderby \& Petheram, 2000; Klop, 1998), although direct comparisons could not be drawn as some studies did not include Audiology clients and some were based only on paediatric populations. This trend, however, becomes clearer when one considers gender in relation to the different disorders.

As found in the present study, as well as other studies (Coyle, Weinrich \& Stemple, 2001), voice disorders are more prevalent amongst females than males. This study found that a large percentage of clients $(n=180 ; 21 \%)$ attended the OPD for the management of a voice disorder and because many of these clients were female the number of females attending the SLT \& Audiology OPD was increased.

There are two possible explanations as to why there were more female than male language disorders found in this study. Firstly, more males presented with language disorders coupled with learning disorders and were thus categorised as such. Secondly, it was found that more of the language disorders fell into the 0-6 year old category. There is a perception that "boys develop more slowly' or 'boys are slower to talk' (Phillips, 2004:500) and they might therefore be brought later for therapy, when they would more likely be diagnosed as having a language and learning difficulty.

Of all the other disorders (apart from language disorders) found mostly in children, all had higher percentages of males than females. Many studies have commented on the greater likelihood of reading difficulties or dyslexia in males than in females (Owens, 1999) and that children with dyslexia have delayed language development. Also, as expected, more males than females attended the OPD for the intervention of fluency disorders (Guitar, 1998).

\section{Home Language}

The majority of clients attending the OPD were classified as having English or Afrikaans as their home language, with a minority speaking Xhosa or another language (Table 2).

Table 2: Number and percentage of clients per Language group who attended the Speech-Language Therapy and Audiology OPD at a tertiary hospital in the Western Cape from 1999-2003 ( $\mathrm{n}=910$ )

\begin{tabular}{|l|c|c|}
\hline Language for Therapy & Number & Percent \\
\hline English & 501 & 55.05 \\
Afrikaans & 298 & 32.75 \\
Xhosa & 82 & 9.01 \\
Other & 18 & 1.98 \\
Missing data & 11 & 1.21 \\
Total & 910 & 100.0 \\
\hline
\end{tabular}

This is interesting considering that $55 \%$ of the population in the Western Cape speak Afrikaans as a home language, 24\% speak Xhosa as a home language and $19 \%$ speak English as a home lan. guage (South Africa.info reporter, 2002). There are several possible reasons for the mismatch between the population profile and the profile of clients attending the SLT \& Audiology OPD.

Firstly, English may be the preferred language of intervention for several reasons. The majority of Speech-Language Therapists and Audiologists in the SLT \& Audiology OPD at this tertiary hospital in the Western Cape were English first language speaking with Afrikaans as a second language. Also, many children were being schooled in English, as English was the language most commonly used as the medium of instruction (Alant, 1989). Thus they may choose to receive therapy in English. Thirdly, there were no Xhosa-speaking Speech-Language Therapists or Audiologists at the study hospital and no formal access to interpreters, resulting in limited provision of services to the Xhosa-peaking population. This situation is of grave concern given the multilingual nature of the population in the region.

Another possible reason for Xhosa-speaking clients not accessing services in the OPD clinic may be due to cultural reasons. Cultural groups vary in their view of disability and therapeutic interventions and such differences may impact who is likely to attend the hospital (Swartz, 1998; Swanepoel, 2005). Furthermore, the hospital in the present study has historically been a 'white' hospital, and there may still be misconceptions about who can access services at this hospital. In many communities in South Africa there is little awareness of SLT \& Audiology services, due to lack of resources and facilities, and therefore people are unaware that services are available.

Access to services is also influenced by socioeconomic status of people. Although poverty is not confined to one racial group in South Africa, it is most prevalent amongst the black population (Swanepoel, 2005). Many of the Xhosa-speaking population in the Western Cape, the majority of whom would be black, may have a limited income. The costs of travel to the hospital as well as multiple treatment fees may be prohibitive.

\section{Geographical Location}

The Health Districts in Cape Town were used to categorise the areas from where the clients who attended the OPDI came.

Table 3: Number and percentage of clients per Geographical location who attended the Speech-Language Therapy and Audiology OPD at a tertiary hospital in the Western Cape from 1999-2003 (n=910)

\begin{tabular}{|l|c|c|}
\hline Geographical Location & Number & Percent \\
\hline Central & 209 & 22.97 \\
Eastern & 22 & 2.42 \\
Khayelitsha & 39 & 4.29 \\
Klipfontein & 258 & 28.35 \\
Mitchells Plain & 117 & 12.86 \\
Northern Panorama & 46 & 5.05 \\
Southern & 150 & 16.48 \\
Tygerberg & 39 & 4.29 \\
Outside Cape Town & 21 & 2.31 \\
Missing data & 9 & 0.99 \\
Total & 910 & 100 \\
\hline
\end{tabular}


Table 3 shows the number and percentage of clients from each area. It can be seen that clients attended from many different areas. This is not surprising given the lack of SLT \& Audiology services in the community or even at a secondary level of care (Allied Health Professionals Technical Committee, 2004). Some of the areas, however, would have been covered by SLT \& Audiology services at another tertiary hospital in Cape Town.

\section{Attendance}

The majority of clients attended the OPD over a very short period of time, for example many attended within a three month period $(n=513 ; 71 \%)$ or attended two appointments $(n=506$; $70 \%$ ). Number of appointments and time frame results showed very similar trends, with a drop-off with increasing number of scheduled appointments or longer period of time over which these appointments were scheduled. Almost half of all the clients $(n=353 ; 49 \%)$ attended only one appointment. Only $9 \%$ $(n=67)$ of clients attended more than five appointments. Given that long-term therapy was often indicated, these findings were noteworthy in relation to service planning.

The high number of clients who only had one booked appointment may be due to the fact that certain clients would have been placed on a waiting list for therapy and therefore not been given further scheduled appointments immedi-

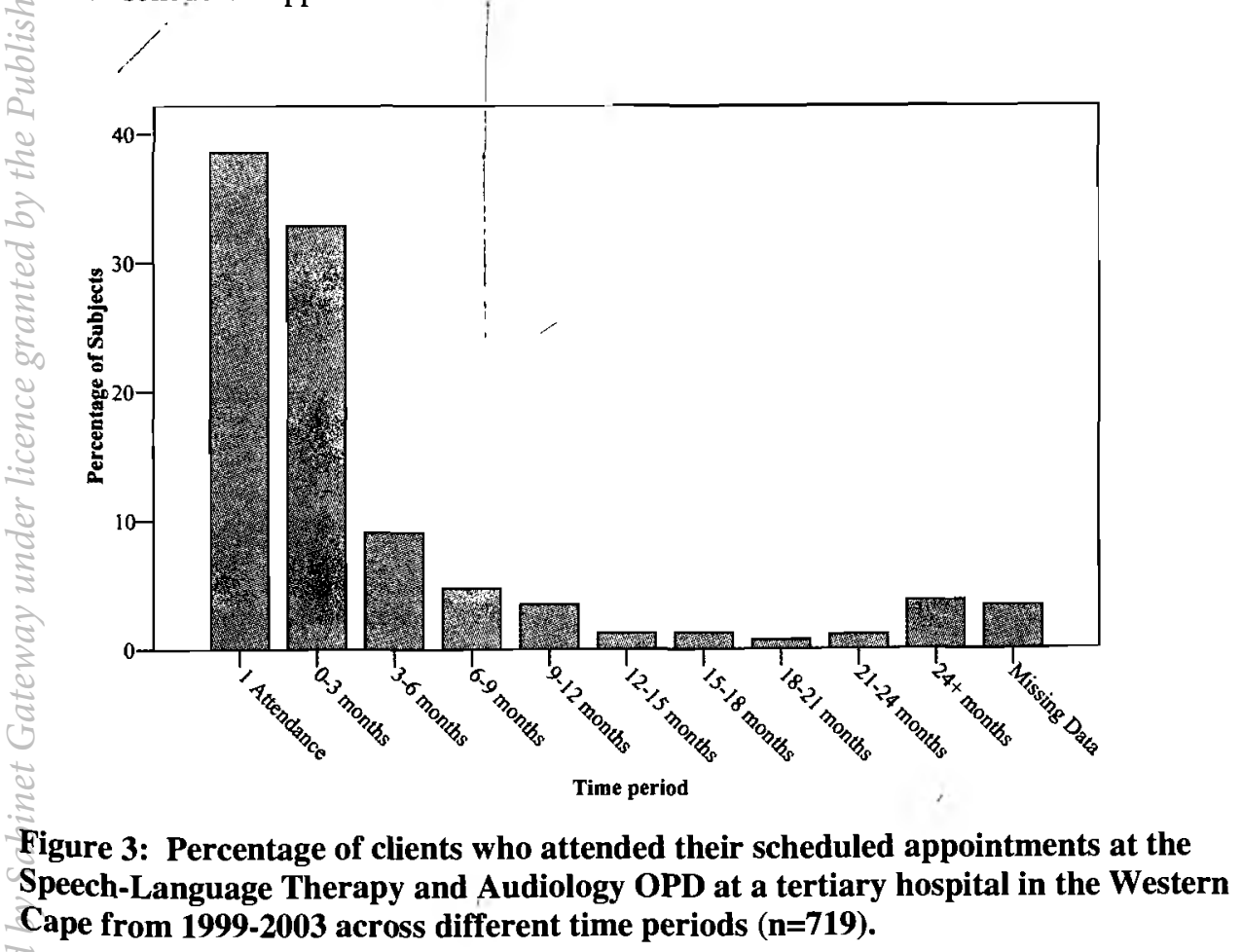

Figure 3: Percentage of clients who attended their scheduled appointments at the Speech-Language Therapy and Audiology OPD at a tertiary hospital in the Western Cape from 1999-2003 across different time periods $(n=719)$.

ately. They may then not have wanted therapy when it was offered, due to changes in circumstances between the time of the assessment and the date when therapy was offered. Additionally, there are some cases for which one attendance may be all that is indicated. Some clients may have needed an initial assessment with no necessary follow-up. It is likely that once their concerns had been addressed, they no longer felt the need for further intervention. Enderby and Davies (1989) reported that $60 \%$ of newly referred children required assessment and advice only. While this explanation might be applicable to some cases, the data in case files, the nature of the disorders and personal experience suggest that many required additional intervention.

The lack of understanding of the nature of interventions offered by the profession of SLT \& Audiology by the community, as well as the location of the services within a tertiary hospital environment, may have contributed to poor attendance rates. Many clients have the expectation that a hospital is a place which one attends when ill and that it is a once-off attendance, possibly with a follow-up appointment. Many expect treatment that is tangible, such as medication or surgery. Clients arrive with the expectation that they will be given something to 'cure' them in a once-off appointment. Therefore, they may not have been prepared for the active role that is required of them in the therapeutic process. Also, they may have been unwilling or unable to commit themselves to fur- ther intervention as recommended. The high rate of non-attendance may also reflect the economically disadvantaged nature of the population served, and the low priority given to SLT \& Audiology services given other life circumstances (Broomfield \& Dodd, 2004).

A further explanation for low attendance rates could relate to different perceptions of a disorder by a therapist and a client, or the client having a different understanding of the nature of a disorder. Negotiating between explanations of illness increases the possibilities of compliance or adherence to treatment (Swartz, 1998). Thus, explaining the nature of the diagnosis in ways that the client can understand and relate to their understanding of the world, may improve attendance and compliance to therapy. Also, discussions prior to commencing therapy about the nature of therapy and the respective roles of the parent and the therapist may well increase parent satisfaction (Roulstone, Glogowska, Peters \& Enderby, 2004), which may improve attendance rates for children. The same principle could be applied to adults.

With an increasing number of scheduled appointments, percentage of appointments attended dropped. The only diagnostic categories which had more than $10 \%$ of clients attending in excess of five appointments were for the categories of fluency disorders ( $n=15$; $28 \%$ ), paediatrics with multiple difficulties $(\mathrm{n}=12 ; 25 \%)$, language disorders $(\mathrm{n}=5 ; 24 \%)$ and phonology/ articulation/ oral motor disorders $(\mathrm{n}=3 ; 13 \%)$. It is possible that the motivation for treatment of these disorders may have been greater than for other disorders.

Data presented by Enderby and Davies (1989), which included many but not all types of communication disorders included in this study, indicated that the children who received regular therapy, attended once a week for an average of 16 weeks. Roulstone et al. (2004) found that of the 71 children allocated to Speech-Language Therapy in their study, 68 attended sessions offered, although nearly $18 \%$ of appointments were either cancelled (by the therapist or clinician) or not attended. Klop (1998) in her private practice found that only two out of 64 clients dropped out of therapy. The differences in findings between these studies confirm the importance of understanding how contextual realities influence service provision. 


\section{LIMITATIONS}

There are several limitations of this study which need to be considered. There has been minimal research into client profiles attending SLT \& Audiology services in South Africa. Therefore, the researcher had to rely on the methodology and literature from other countries and other healthcare disciplines to conduct this study. While such literature was useful, it was not directly applicable to the SLT \& Audiology contexts in South Africa. The study was also limited to the data from one tertiary hospital examined over a five year time period. The conclusions therefore are only applicable to the tertiary hospital being studied during the specified time period.

Due to the nature of the study, records used were not devised specifically for research purposes and thus may not have been maintained with the care that would be expected in a planned investigation. Notably, as diagnostic labels vary in use across SLT \& Audiology services and medical categories, specific diagnoses are open to different interpretations. For example, a patient's primary medical diagnoses may be a "cerebral-vascular accident" with SLT \& Audiology services referring to "neurogenic acquired communication disorders". This resulted in difficulties during the data collection phase which included missing records, incomplete records, and information recorded in an inconsistent manner within and between records. This is a common limitation of retrospective studies such as those by Farmer (1990) and Schneider (1992). A further problem associated with the description of communication disorders is that many disorders (for example learning difficulties) are not clearly defined (Lubker, 1997).

Limitations in the interpretation of the results included lack of comparison data from other sites, limited ability to generalize the findings of this research to other service sites, and that the interpretation was speculative in nature. Due to the descriptive nature of the study, no causal or correlational assertions could be drawn (Hite, 2001). Despite such limitations, the findings can inform the restructuring of services in the region.

The difficulties encountered with the use of records points to the need for good record keeping, which is essential for planning of healthcare services (Rhoda, 2001). Inadequate records can distort research results, prevent clients from being involved in research and negate the quality of research (Farmer, 1990). Therefore, methods of recording client information and treatments used need to be reviewed (Farmer, 1990). This information is not only useful for research, but also when assessing cost-efficiency of a service (Rhoda, 2001).

The limited access to SLT \& Audiology services in the Western Cape, as reflected in the poor attendance rates obtained in this study, point to the need for SLT \& Audiology services at all levels, and particularly at a primary level, of care (Rhoda, 2001). Services need to be provided in line with the Primary Health Care Approach within the District Health System (Hall et al., 2002; Forman, et al., 2004). Ideally, the services should be integrated at all levels of care and should strive towards community based rehabilitation. As a starting point, therapists' participation in community outreach programmes would be advantageous.

\section{CONCLUSION}

This study found that the highest percentages of clients attending the SLT \& Audiology OPD at a tertiary hospital in the Western Cape attended for a hearing loss $(n=250 ; 29 \%)$ or voice disorder $(\mathrm{N}=180 ; 21 \%)$ and that the ENT department at the study hospital $(n=203 ; 24 \%)$ and the education sector $(n=111 ; 13 \%)$ re- ferred the highest percentages of clients. In terms of age, the highest percentage of clients attending the OPD were between 7 and 12 years old $(n=165 ; 18 \%)$. Overall there were similar percentages of male and female clients who attended the OPD. English speaking clients predominated $(\mathrm{n}=501 ; 55 \%)$, followed by Afrikaans speaking clients $(n=298 ; 33 \%)$. Only a small percentage of clients were Xhosa speaking $(n=82 ; 9 \%)$. Patterns of attendance showed that almost half of all the clients who attended the OPD attended only one appointment ( $\mathrm{n}=353 ; 49 \%)$. In total just over $70 \%$ of clients attended either no more than two appointments $(n=506 ; 70 \%)$ or attended within a three month period $(n=513 ; 71 \%)$. With an increasing number of scheduled appointments, percentage of appointments attended decreased.

Given the poor attendance rates and the high number of clients in the 7 to 12 year age group seen at the SLT \& Audiology $\mathrm{OPD}$, there is a definite need for more adequate service provision in schools. The Department of Education in the Western Cape has very limited SLT \& Audiology services (F. Lewis, personal communication, February 18, 2005). However, the Department of Education (2001) emphasises the need to optimize the expertise of specialist support personnel, such as therapists, psychologists, remedial educators and health professionals within the school setting. In the long term therefore, the service provision for learners in inclusive settings must be given urgent consideration. Currently, the Education sector in the Western Cape does not have the capacity for individual intervention in ordinary classrooms, but plans to provide preventative services and services to educators (F. Lewis, personal communication, February 18, 2005).

One of the major challenges facing the SLT \& Audiology profession is to provide equitable services for the multilingual populations. The scarcity of Xhosa-speaking therapists in the Western Cape is a source of concern. In the long term it would be advantageous to train and employ professionals speaking African languages. However, given the language diversity in the country, it is critical that practitioners become skilled to manage multilingual and multicultural populations (Swanepoel, 2005). For example, the inclusion of interpreters to support clinical practice is important. However, there were no available interpreters at the SLT \& Audiology OPD under study, despite repeated requests to the hospital authorities in this regard. While service delivery to multilingual populations remains a challenge, it is important that steps are taken to create equitable practices.

\section{REFERENCES}

Abramson, J. (1990). Survey methods in community medicine. Edinburgh: Churchill Livingstone.

Alant, E. (1989). Language intervention at schools: Changing orientations within the South African context. The South African Journal of Communication Disorders, 36: 9-13.

Allied Health Professionals Technical Committee (February 2004). Rehabilitation 2010: Draft 1.

Bhagwanjee, A., \& Stewart, R. (1999). Disability research in South Africa: vision and imperatives for a national coordinated approach. South African Journal of Occupational Therapy, 29(1): 15-17.

Blake, R. (1981). Disabled older persons: A demographic analysis. Journal of Rehabilitation, 47(4), 19-27.

Broomfield, J., \& Dodd, B. (2004). Children with speech and language disability: Caseload characteristics. International Journal of Language and Communication Disorders, 39(3): 303-324. 
Council for International Organisations of Medical Sciences [CIOMS] (1991). International Guidelines for ethical reviews of epidemiologic studies. Geneva: CIOMS,.

Coyle, S., Weinrich, B., \& Stemple, J. (2001). Shifts in relative prevalence of laryngeal pathology in a treatment-seeking population. Journal of Voice, 15(3): 424-440.

Das, J. (2001). Reading Difficulties \& Dyslexia. New Jersey: Sarka Educational Resources Inc.

Department of Education (2001). White Paper 6: Special Education: Building an inclusive education and training system. Pretoria: Government Printer.

Edwards, M., Cape, J., \& Brown, D. (1989). Patterns of referral for children with speech disorders. Child: Care, Health and Development, 15: 417-424.

Enderby, P. (1989). The epidemiology of communication disorders. In M. Leahy (Ed.), Disorders of Communication: The Science of Intervention. London: Taylor and Francis.

Enderby, P., \& Davies, P. (1989). Communication disorders: Planning a service to meet the needs. British Journal of Disorders of Communication, 24: 301-331.

Enderby, P., \& Petheram, B. (2000). An analysis of referrals to speech and language therapy in 11 centres, 1987-95. International Journal of Language and Communication Disorders, 35(1): 137-146.

Farmer, H. (1990). Can retrospective research be done from records? S.A. Journal of Occupational Therapy, 20: 18-21.

Forman, L., Pillay, Y., \& Sait, L. (2004). Health Legislation 19942003. In Health Systems Trust, South African Health Review 2003/04. Durban: Health Systems Trust.

Guitar, B. (1998). Stuttering: An Integrated Approach to its Nature and Treatment (second edition). Maryland, Baltimore: Lippincott Williams and Wilkins.

Hall, W., Haynes, R., \& McCoy, D. (2002). The Long Road to the District Health System: Legislation and Structures for the DHS in South Africa: An Appraisal as at August 2002. Durban: Health Systems Trust.

Heron, C. (2001). Speech and Language Therapy for Adults in Hackney: An Assessment of Need. International Journal of Communication Disorders, 36(suppl): 46-51.

Hite, S. (2001). Reviewing Quantitative Research to Inform Educational Policy Processes. Paris: UNESCO: International Institute for Educational Planning.

Jumba, P., \& Day, C. (2004). Preface. In Health Systems Trust, South African Health Review 2003/04. Durban: Health Systems Trust.

Katzenellenbogen, J., Joubert, G., \& Abdool Karim, S. (Eds). (1997). Epidemiology: A Manual for South Africa. Cape Town: Oxford University Press.

Klop, D. (1998). Quality management in a private Speech-Language Therapy practice. Unpublished M.Sc. (Speech and Language Pathology) thesis, University of Cape Town.

Lubker, B. (1997). Epidemiology: An essential science for speechlanguage pathology and audiology. Journal of Communication Disorders, 30: 251-267.
Lubker, B., \& Tomblin, J. (1998). Epidemiology: Informing clinical practice and research on language disorders of children. Topics in Language Disorders, 19(1): 1-26.

McLaren, P., Solarsh, G., \& Saloojee, G. (2004). Disability and disablement. In Health Systems Trust, South African Health Review 2003/04. Durban: Health Systems Trust.

Ntuli, A., \& Day, C. (2004). Ten years on - Have we got what we ordered? In Health Systems Trust, South African Health Review 2003/04. Durban: Health Systems Trust.

Owens, R. (1999). Language Disorders: A Functional approach to assessment and intervention (third edition). Boston: Allyn and Bacon.

Petheram, B., \& Enderby, P. (2001). Demographic and epidemiological analysis of patients referred to speech and language therapy at eleven centres 1987-95. International Journal of Language and Communication Disorders, 36(4): 515-525.

Phillips, J. (2004). Language delay: Why do parents fail appointments? International Journal of Therapy and Rehabilitation, 11(11): 500 .

Pillay, M., Kathard, H., \& Samuel, M. (1997). The curriculum of practice: A conceptual framework for Speech-Language Therapy and Audiology practice with a Black African first language clientele. The South African Journal of Communication Disorders, 44: 109-117.

Raia, P. (1992). What are we trying to restore? A case for habilitation. Generations, 16(1): 37-39.

Rhoda, A. (2001). A profile of stroke clients treated at the Bishop Lavis Rehabilitation Centre between 1995-1999. Unpublished Master of Medical Sciences (Rehabilitation) thesis, University of Stellenbosch.

Roulstone, S., Glogowska, M., Peters, T., \& Enderby, P. (2004). Building good practice: lessons from a multimethod study of speech and language therapy. International Journal of Therapy and Rehabilitation, 11(5): 199-204.

Schneider, M. (1992). The nature and management of communication disorders in a rural area: The role of the Community Speech and Hearing Therapy Workers. South African Journal of Communication Disorders, 39: 55-61.

South Africa info reporter. (2002). SA's population by province. Retrieved February 21, 2005, from http://www.southafrica.info/ ess_info/sa_glance/demographics/popprov.htm

Statistics South Africa (2004). Retrieved June 22, 2004, from SuperCross. Space Time Research www.info.gov.za/ annualreport/2003/statssa03.pdf

Statistical Package for the Social Sciences Inc. (1995). Statistical Package for the Social Sciences. Chicago, Illinois: SPSS Inc.

Swanepoel, D. (2005). Infant Hearing Screening at Maternal and Child Health Clinics in a developing South African community. University of Pretoria: Unpublished DPhil dissertation.

Swartz, L. (1998). Culture and mental health: A Southern African view. Cape Town: Oxford University Press.

Whitelaw, D., Meyer, C., Bawa, S., \& Jennings, K. (1994). Postdischarge follow-up of stroke patients at Groote Schuur Hospital - A prospective study. South African Medical Journal, $84: 11-13$ 


\section{APPENDIX A}

\section{Data Collection Form}

Demographic Information - Date in file

Client's Name:

Date of Birth:

Full Residential Address:

Monthly Family Income:

Hospital category: Free Ho H1 H2 H3 Private

Marital Status:

Occupation: Pensioner

Grant
Folder number:

Gender: Male Female

Home language:

\section{Diagnostic information}

Medical Diagnosis:

Speech/Audio Diagnosis:

Referral source:

Date assessed:

\section{Attendance information (write every date)}

Dates attended therapy sessions:

Dates cancelled therapy sessions:

Dates of appointments missed: 